\title{
Topological Transition of Superconductivity in Dirac Semimetal Nanowire Josephson Junctions
}

\author{
Cai-Zhen Li, ${ }^{1,2, *}$ An-Qi Wang, ${ }^{2,3, *}$ Chuan Li, ${ }^{4, \dagger}$ Wen-Zhuang Zheng, ${ }^{2}$ Alexander Brinkman, ${ }^{4}$ \\ Da-Peng Yu, ${ }^{1}$ and Zhi-Min Liao $\circledast^{2,5, *}$ \\ ${ }^{1}$ Shenzhen Institute for Quantum Science and Engineering, and Department of Physics, \\ Southern University of Science and Technology, Shenzhen 518055, China \\ ${ }^{2}$ State Key Laboratory for Mesoscopic Physics and Frontiers Science Center for Nano-optoelectronics, School of Physics, \\ Peking University, Beijing 100871, China \\ ${ }^{3}$ Academy for Advanced Interdisciplinary Studies, Peking University, Beijing 100871, China \\ ${ }^{4}$ MESA+ Institute for Nanotechnology, University of Twente, 7500 AE Enschede, The Netherlands \\ ${ }^{5}$ Beijing Key Laboratory of Quantum Devices, Peking University, Beijing 100871, China
}

(Received 20 October 2020; accepted 18 December 2020; published 12 January 2021)

\begin{abstract}
We report the topological transition by gate control in a $\mathrm{Cd}_{3} \mathrm{As}_{2}$ Dirac semimetal nanowire Josephson junction with diameter of about $64 \mathrm{~nm}$. In the electron branch, the quantum confinement effect enforces the surface band into a series of gapped subbands and thus nontopological states. In the hole branch, however, because the hole mean free path is smaller than the nanowire perimeter, the quantum confinement effect is inoperative and the topological property maintained. The superconductivity is enhanced by gate tuning from electron to hole conduction, manifested by a larger critical supercurrent and a larger critical magnetic field, which is attributed to the topological transition from gapped surface subbands to a gapless surface band. The gate-controlled topological transition of superconductivity should be valuable for manipulation of Majorana zero modes, providing a platform for future compatible and scalable design of topological qubits.
\end{abstract}

DOI: 10.1103/PhysRevLett.126.027001

Much effort has been paid to find routes for the realization of topological superconductivity in several platforms, ranging from the presumably intrinsic topological superconductors [1-3], topological materials [4], and semiconductor nanowires $[5,6]$ proximitized with an $s$-wave superconductor, ferromagnetic atomic chains $[7,8]$, and iron-based superconductors [9-12], etc. The topological superconducting phase transition has been obtained by applying a magnetic field in semiconductor nanowires coupled with a superconductor, showing the signatures of Majorana fermions [13-15]. Recently, topological transitions have also been proposed based on Josephson junctions in a 2D electron gas [16-19], which can be tuned by a superconducting phase difference. In addition, the topological superconductivity can be realized in the topological surface and edge states proximitized with a superconductor [4,20-28].

The topological transition of topological materials themselves will trigger the phase transition of the proximityinduced topological superconductivity accordingly. Such a topological phase transition can be realized by magneticfield-induced opening and closing of the Dirac gap [29,30], electric-field control [31-33], applying strain or high pressure [34-38], etc. In addition, the quantum confinement also provides a way to induce discrete surface subbands in thin nanowires. Tuning the quantum confinement will tune the phase transition with opening and closing of the surface Dirac gap, accompanied by the presence or absence of quantized surface subbands. Combining the topological transition with superconductivity opens an avenue for fine control of Majorana zero modes.

Here we report the topological transition of superconductivity in $\mathrm{Cd}_{3} \mathrm{As}_{2}$ Dirac semimetal nanowire-based Josephson junctions by gate control [Fig. 1(a)]. The $\mathrm{Cd}_{3} \mathrm{As}_{2}$ nanowires were grown by chemical vapor deposition method [39], which are of single crystalline nature and grown along the [110] direction (see Supplemental Material, Fig. S1 [40]). The topological transition of $\mathrm{Cd}_{3} \mathrm{As}_{2}$ surface states is revealed by quantum oscillations under a magnetic field parallel to the nanowire axis. Because of the relatively high electron mobility and low hole mobility in $\mathrm{Cd}_{3} \mathrm{As}_{2}$ [43-45], the quantum confinement effect could be notable for the electron conduction and negligible for hole conduction in a thin nanowire with diameter of $\sim 64 \mathrm{~nm}$ in this Letter [Fig. 1(b)]. Because the mean free path of electrons is comparable with the nanowire perimeter, Aharonov-Bohm (AB) oscillations with flux quantum $(h / e)$ periodicity are expected $[46,47]$. Whereas, for the diffusive transport of holes, the Altshuler-Aronov-Spivak (AAS) oscillations with $h / 2 e$ period become prominent. A clear transition from $h / e$ period $\mathrm{AB}$ oscillations $(\Delta B=1.28 \mathrm{~T})$ to $h / 2 e$ period AAS 
(a)

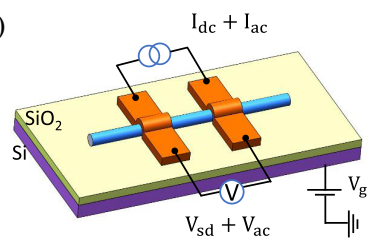

(b)

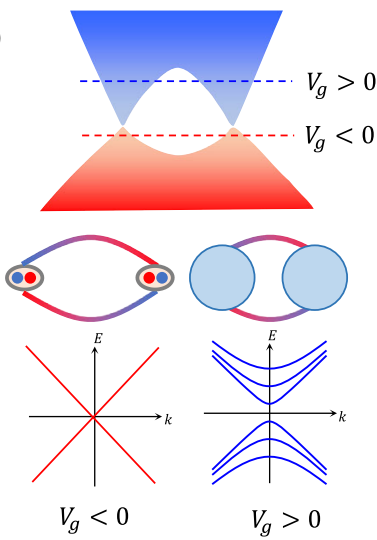

(c)

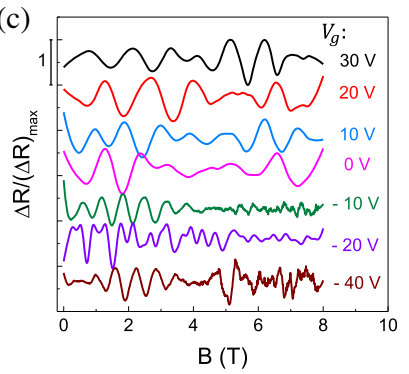

(d)

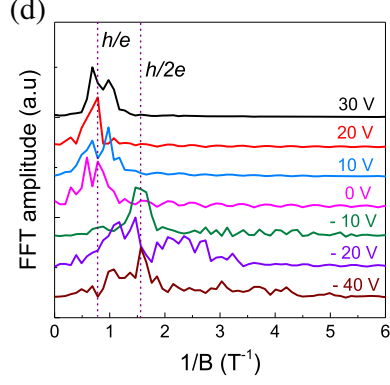

FIG. 1. Normal state transport in $\mathrm{Nb}-\mathrm{Cd}_{3} \mathrm{As}_{2}-\mathrm{Nb}$ junction. (a) Schematic of the measurement setup. The junction length is $\sim 400 \mathrm{~nm}$. (b) Top: the bulk bands schematic diagram of $\mathrm{Cd}_{3} \mathrm{As}_{2}$. Middle: the schematic of Fermi arc surface states. Bottom: the continuous Dirac surface band for $V_{g}<0$ and quantized surface subbands due to quantum confinement effect for $V_{g}>0$. (c) The normalized resistance oscillations $\Delta R /(\Delta R)_{\max }$ after subtracting the background. The magnetic field is applied in plane along the nanowire axis. (d) FFT spectrums of the resistance oscillations under different gate voltages.

oscillations $(\Delta B=0.64 \mathrm{~T})$ is observed by tuning the gate voltage from positive to negative [Figs. 1(c) and 1(d); the raw data in Fig. S2 [40] ], indicating a transition from gapped quantized surface subbands to a gapless surface band.

We then focus on the superconducting behavior with and without the quantized surface subbands at a base temperature of $12 \mathrm{mK}$. Figure 2(a) shows the current-voltage $\left(I_{\mathrm{dc}}-V_{\mathrm{sd}}\right)$ characteristics of the junction. The superconducting state shows a critical current $I_{c}$ varying with $V_{g}$. The differential resistance $d V / d I$ as a function of $V_{g}$ and $I_{\mathrm{dc}}$ is presented in Fig. 2(b), and the $I_{c}$ is marked by the solid white line. As sweeping $V_{g}$ from 18 to $-30 \mathrm{~V}$, the $I_{c}$ decreases first and then tends to saturate in the hole region. The small and saturated $I_{c}$ in the hole region is due to the low hole mobility, where the supercurrent through bulk states is significantly suppressed $[25,48]$. An excess current $I_{\text {exc }} \sim 210 \mathrm{nA}$ in the $I-V$ characteristic gives a transparency $D \sim 0.73$ of the junction interface [49] (Supplemental Material, Fig. S3 [40]).

An in-plane magnetic field $B$ is applied along the nanowire axis. Figure 2(c) shows the $d V / d I$ as a function of $B$ and $I_{\mathrm{dc}}$ at $V_{g}=0 \mathrm{~V}$. From 0 to $250 \mathrm{mT}, I_{c}$ is strongly suppressed from 180 to $3 \mathrm{nA}$. Beyond the initial fast decay,

the $I_{c}(B)$ exhibits an irregular oscillation behavior with multiple nodes, which can be seen up to 2 T. Figures 2(d)2(f) show the enlarged $I_{c}(B)$ oscillations at three different gate voltages. The $I_{c}(B)$ exhibits multiple nodes at $V_{g}=22$ and $0 \mathrm{~V}$ [Figs. 2(d) and 2(e)], while only two nodes at $V_{g}=-30 \mathrm{~V}$ [Fig. 2(f)]. The successive evolution of $I_{c}(B)$ oscillations as a function of $V_{g}$ is displayed in Fig. $2(\mathrm{~g})$. For positive $V_{g}$, the $I_{c}(B)$ shows multiple nodes with maxima and minima sensitively depending on $V_{g}$. For negative $V_{g}$, only two nodes are observed and the period is gate independent. Note that the $I_{c}(B)$ oscillatory behavior can only be observed when the magnetic field is applied along the nanowire axis. While under an in-plane magnetic field perpendicular to the nanowire axis, the $I_{c}(B)$ exhibits a monotonic decay without any oscillations (Supplemental Material, Fig. S4 [40]).

This distinct gate dependence of the $I_{c}(B)$ oscillatory behavior can be well explained by considering the presence (absence) of quantized surface subbands at positive (negative) $V_{g}$. For positive $V_{g}$ at zero magnetic field, it is natural that the supercurrent is from the coexisting bulk and surface states due to the highly conductive bulk and large surface-to-volume ratio in $\mathrm{Cd}_{3} \mathrm{As}_{2}$ nanowires. When applying a magnetic field, the bulk carried supercurrent is strongly suppressed (as indicated by the $I_{c}$ kink behavior at around $0.15 \mathrm{~T}$ in Fig. 2(i); also see Supplemental Material, Figs. S5 and S6 [40]), while the supercurrent carried by surface states can survive to high magnetic fields [50]. So, the $I_{c}$ oscillations under high magnetic fields should be attributed to the quantized surface subbands.

Below, we illustrate how the quantized surface subbands result in $I_{c}$ oscillations. The quantized surface subbands can be described by the angular momentum quantum number $l[30,47]$. In the presence of a parallel magnetic field, the motion of particles on the nanowire surface travels in a spiraled path when they traverse the junction from one $\mathrm{Nb}$ electrode to the other [Fig. 2(h)]. Different surface modes with different quantized angular momentum $l$ traverse a different path along the nanowire and thus acquire a different orbital phase $\Delta \phi=(2 e / \hbar)$ $\int_{\theta_{1}}^{\theta_{2}} \vec{A} \cdot R d \theta_{l}$, where $\vec{A}$ is the vector potential, $R$ is the radius, and $\theta_{l}$ is the corresponding winding angle. The quantum interference between supercurrents carried by different surface modes consequently leads to the multiple $I_{c}$ oscillation nodes [51-53]. Since the occupation of surface modes can be modulated by a gate voltage, the oscillation nodes are gate sensitive. The red line in Fig. 2(d) is the numerical simulation of supercurrent interference with occupied surface modes $|l| \leq 8$ (see Supplemental Material, Figs. S5 and S6 [40] for more details). The occupied multiple orbital modes with $|l| \leq 8$ at $V_{g}=22 \mathrm{~V}$ are roughly consistent with the allowed number of modes in the surface states. For a carrier density $n_{c}$ of the order of $10^{18} \mathrm{~cm}^{-3}$, the corresponding Fermi wave 

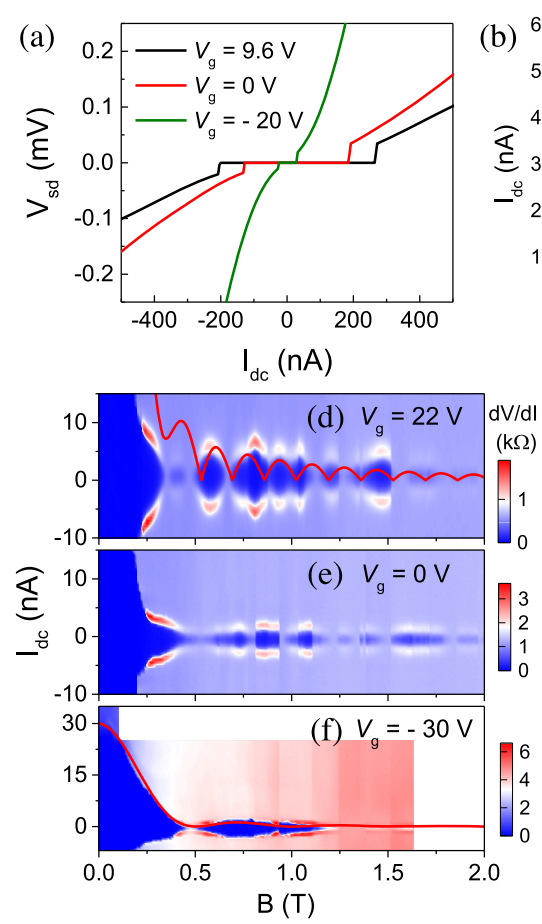
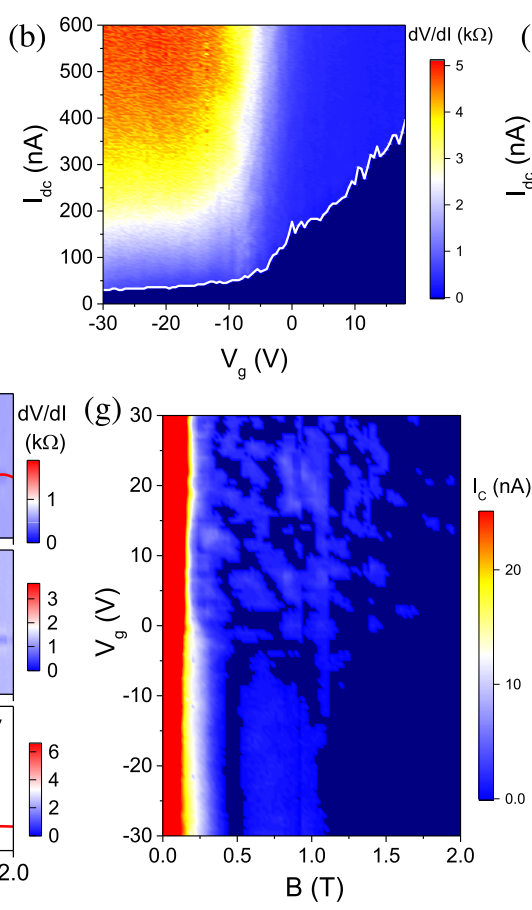

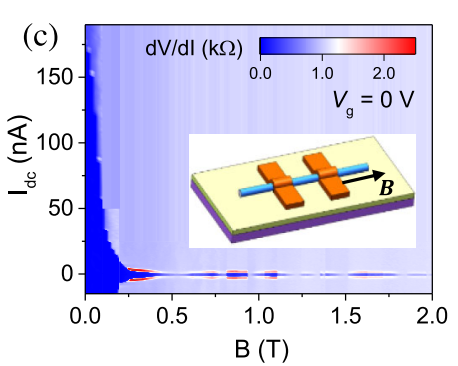

(h)
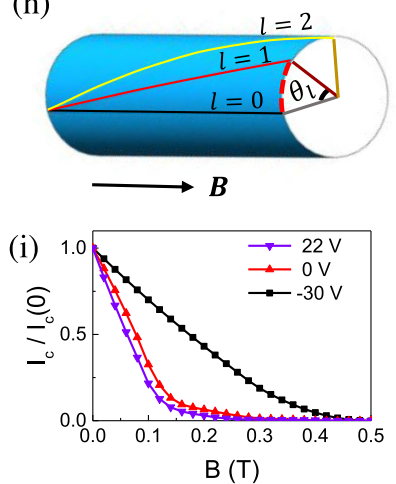

FIG. 2. The supercurrent and $I_{c}$ evolution with the axial magnetic field. (a) The $V_{\mathrm{sd}}$ versus $I_{\mathrm{dc}}$ characteristics. (b) Differential resistance $d V / d I$ as a function of $I_{\mathrm{dc}}$ and $V_{g}$. An excitation of $I_{\mathrm{ac}}=0.5 \mathrm{nA}$ was used for the $d V / d I$ measurement. The solid white line marks the critical current $I_{c}$ varying with $V_{g}$. (c) Mapping of $d V / d I$ as a function of $I_{\mathrm{dc}}$ and magnetic field at $V_{g}=0 \mathrm{~V}$. The $I_{\mathrm{dc}}$ is swept from negative to positive. Inset: schematic of the $\mathrm{Nb}-\mathrm{Cd}_{3} \mathrm{As}_{2}-\mathrm{Nb}$ junction with an in-plane magnetic field along the nanowire direction. (d)-(f) The detailed patterns of $d V / d I$ as a function of $I_{\mathrm{dc}}$ and magnetic field at different $V_{g}$. Red curve in (d): the $I_{c}$ fit by the model of supercurrent interference between different surface modes. Red curve in (f): the $I_{c}$ fit by the model of continuous surface band, with both orbital and Zeeman phase modulation considered. (g) Mapping of critical current $I_{c}$ as a function of $V_{g}$ and magnetic field. (h) Schematic of the supercurrent interference between different surface modes characterized by the angular momentum quantum number $l$. $\theta_{l}$ is the corresponding winding angle. (i) The normalized critical current $I_{c} / I_{c}(0)$ as a function of magnetic field.

vector is $k_{f} \sim 3 \times 10^{8} \mathrm{~m}^{-1}$. Considering that $k_{\theta}=(1 / R)=$ $3 \times 10^{7} \mathrm{~m}^{-1}$, the maximum value of quantum number $l$ is about $\left(k_{f} / k_{\theta}\right) \sim 10$. Therefore, most of the circular modes contribute to the supercurrent. For $V_{g}=0 \mathrm{~V}$, the $I_{c}$ values are very small and cannot be simulated properly, and the oscillation behavior is likely disturbed by the trapping of magnetic vortex, as indicated by the abrupt vertical lines in Fig. 2(e). The presence of vortex trapping also gives a possible origin of the slight deviation between the data and fitting curve in Fig. 2(d).

For $V_{g}=-30 \mathrm{~V}$, the supercurrent is mainly carried by surface states due to the low hole mobility of bulk state. With increasing magnetic field, the suppression rate of $I_{c}$ at $V_{g}=-30 \mathrm{~V}$ is much lower than that at $V_{g}=22$ and $0 \mathrm{~V}$ [Fig. 2(i)] due to the topological protection of surface states. As a surface particle traverses along the perimeter of the nanowire from position $x_{1}$ to $x_{2}$, the phase modulation is given by $\phi_{2}\left(x_{2}\right)-\phi_{1}\left(x_{1}\right)=\left[\pi B\left(x_{2}-x_{1}\right) R / \phi_{0}\right]$, where $\phi_{0}=h / 2 e, \phi_{1}\left(x_{1}\right)$, and $\phi_{2}\left(x_{2}\right)$ are the phases of the order parameters (Supplemental Material, Fig. S7a [40]). As the surface bands are of continuous form without quantization at negative $V_{g}$, the integration of the supercurrent should be over the entire circle of the nanowire, which is expressed as
$I\left(\varphi_{0}, B\right) \sim \int_{0}^{2 \pi R} \int_{0}^{2 \pi R} d x_{1} d x_{2} \sin \left[\varphi_{0}+\phi_{2}\left(x_{2}\right)-\phi_{1}\left(x_{1}\right)\right]$.

The critical current is defined as the maximum in one period of $2 \pi$ phase, $I_{c}(B)=\max \left[I\left(\varphi_{0}, B\right)\right]$. As the surface particles travel through an entire circle, the period of the $I_{c}$ oscillation is expected to be $\phi_{0}(h / 2 e)$, with a magnetic field period $\Delta B=0.64 \mathrm{~T}$ (Supplemental Material, Fig. S7c [40]). However, despite the fact that the normal state $h / 2 e$ periodic AAS oscillations demonstrate a magnetic field period of $0.64 \mathrm{~T}$, the $I_{c}$ oscillation in Fig. 2(f) presents a period $\Delta B=0.5 \mathrm{~T}$. This deviation can be compensated by taking the Zeeman effect into account. When applying a magnetic field along the nanowire axis, the Fermi surface of surface state is shifted along the $x$ direction (along the perimeter) by $\left(g \mu_{B} B / \hbar v_{f}\right)$ due to the helical spinmomentum locking property of the Fermi arc surface states [54] (Supplemental Material, Fig. S7b [40]). As a result of the shift, the Cooper pairs, formed by the electrons on the $\mathrm{Cd}_{3} \mathrm{As}_{2}$ Fermi surface, gain a finite momentum $\Delta k_{x}=\left(2 g \mu_{B} B / \hbar v_{f}\right)$ and, consequently, acquire an extra 

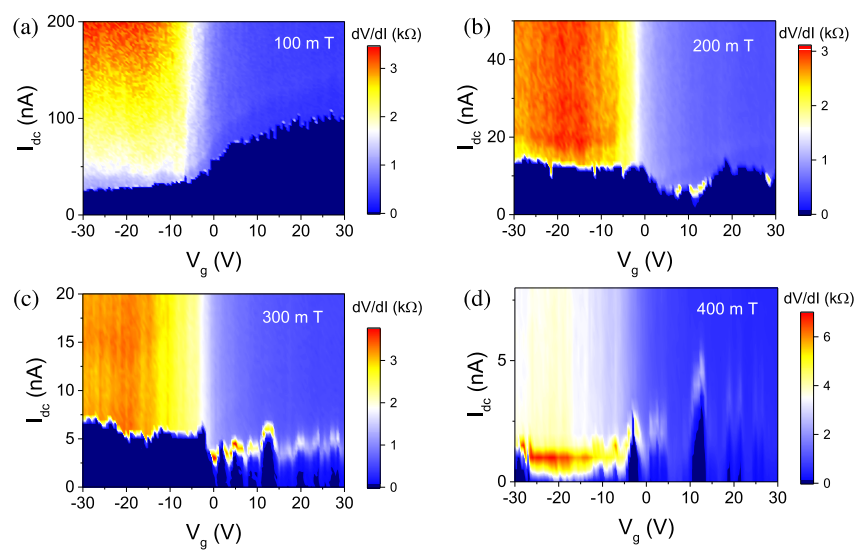

FIG. 3. (a)-(d) Mapping of $d V / d I$ as a function of $I_{\mathrm{dc}}$ and $V_{g}$ at different magnetic fields as denoted. At a moderate field of $300 \mathrm{mT}$, the $I_{c}$ at negative $V_{g}$ is larger than that at positive $V_{g}$.

phase $\Delta k_{x}\left(x_{2}-x_{1}\right)$. Numerically, the experimental $I_{c}$ oscillation at $V_{g}=-30 \mathrm{~V}$ can be well fitted by summing up the orbital and Zeeman phases [red line in Fig. 2(f)]. It is necessary to note that the Zeeman effect itself could not lead to an $I_{c}$ oscillation in the magnetic field range of 0-2 T (Supplemental Material, Fig. S7d [43]). The contribution of the Zeeman effect is small and it just gives a tiny modification to the period of $I_{c}$ oscillation, especially in the regime of multiple surface modes at $V_{g}=22 \mathrm{~V}$ (Supplemental Material, Fig. S8 [40]). In addition, numerical simulations indicate that the orbital interference of bulk states only renders a monotonous $I_{c}$ decay without oscillations (Supplemental Material, Fig. S9 [40]), further confirming the surface origin of $I_{c}$ oscillations under higher magnetic fields.

To further study the gate control of topological phase transition, gate sweeps of the supercurrent under different magnetic fields are performed, as presented in Fig. 3. At a low field of $100 \mathrm{mT}$, because the supercurrent at positive $V_{g}$ is carried by coexisting bulk and surface states, the supercurrent interference from quantized surface subbands is submerged in a large $I_{c}$ background [Fig. 3(a)]. Gradually increasing the magnetic field to suppress the bulk carried supercurrent, the supercurrent interference from quantized surface subbands starts to emerge [Fig. 3(b)]. The gate control of phase transition is obtained, as the bulk carried supercurrent is fully suppressed under $300 \mathrm{mT}$ [Fig. 3(c)]. In this case, with the presence of gapped surface subbands at positive $V_{g}$, the supercurrent interference between different surface modes leads to an apparent $I_{c}$ oscillation behavior as tuning the occupation of surface subbands by $V_{g}$ [Fig. 3(c)]. On tuning the gate voltage to negative, the low mobility of holes leads to a diffusive transport along the perimeter; thus the quantum confinement effect is inoperative and, consequently, gives rise to the preservation of gapless surface band. This is consistent with the disappearance of interference-induced $I_{c}$ oscillations at negative $V_{g}$, where only a nearly flat $I_{c}$ versus $V_{g}$ is
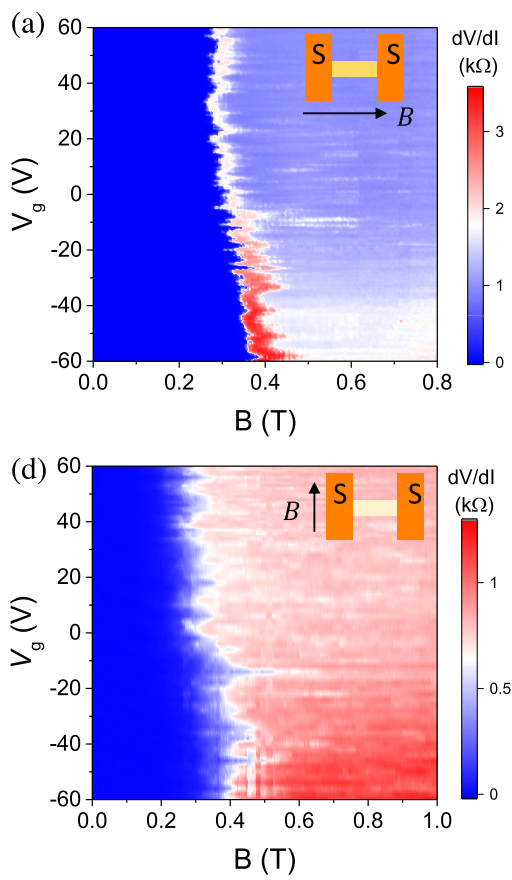
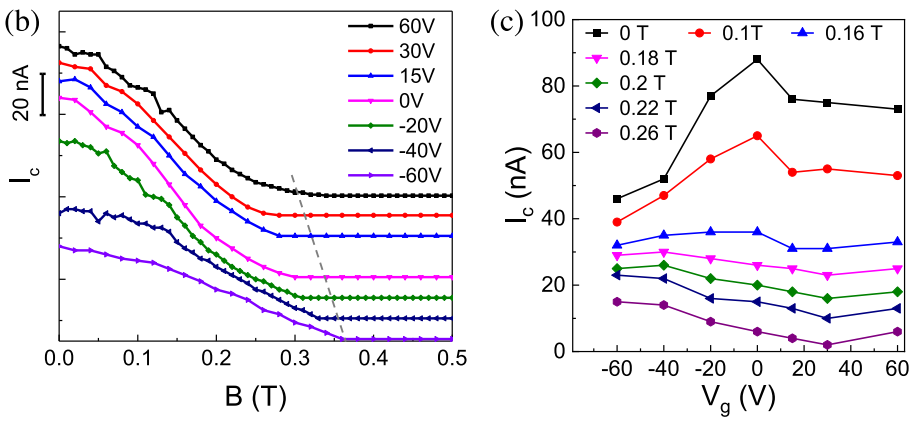

(e)

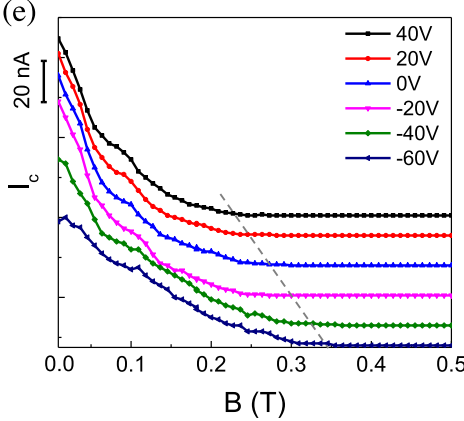

(f)

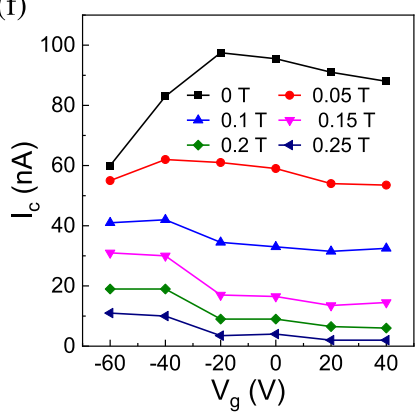

FIG. 4. The proximity-induced superconductivity in device 2 with diameter $d=72 \mathrm{~nm}$ and junction length $L=400 \mathrm{~nm}$. (a) Color map of $d V / d I$ as a function of magnetic field and gate voltage. Inset: the magnetic field direction with respect to the Josephson junction. $S$ denotes superconducting electrodes. (b) The critical current $I_{c}$ versus parallel magnetic field. The curves are shifted for clarity. Gray dotted line indicates the evolution of critical magnetic field $B_{c}$ at which $I_{c}$ decays to zero. (c) The gate dependence of $I_{c}$ at different parallel magnetic fields as denoted. (d)-(f) Similar results under an in-plane perpendicular magnetic field. 
observed. In addition, benefitting from the topological property of the gapless surface band, the supercurrent in this scheme is more robust than that carried by gapped surface modes, as indicated by the larger $I_{c}$ amplitude at negative $V_{g}$ than that at positive $V_{g}$ [Fig. 3(c)].

The negative gate voltage enhanced superconductivity is also observed in a second device (device 2, with a diameter of $72 \mathrm{~nm}$ and junction length $L=400 \mathrm{~nm}$ ). The $I_{c}$ oscillations in device 2 can still be observed but are not as apparent as that in device 1 (Supplemental Material, Figs. S10 and S11 [40]), possibly due to the longer perimeter and weaker superconducting coupling. Nevertheless, it is interesting to find that the critical magnetic field $B_{c}$ is enhanced as tuning the gate voltage from electron to hole conduction [Fig. 4(a)]. The enhanced $B_{c}$ is also observed from the magnetic field dependence of $I_{c}$ at different gate voltages, as the gray dotted line indicated in Fig. 4(b). The slower decay of $I_{c}$ at negative $V_{g}$ is due to the topological protection of surface states. Figure 4(c) exhibits the gate dependence of $I_{c}$ at different magnetic fields. At a moderate magnetic field $\left(B \geq 0.18 \mathrm{~T}\right.$ ), the $I_{c}$ at negative $V_{g}$ is larger than that at positive $V_{g}$, consistent with that shown in Fig. 3(c). Similar $B_{c}$ and $I_{c}$ enhancement is also observed under an in-plane perpendicular magnetic field [Figs. 4(d)-4(f)].

In summary, we have demonstrated the gate-tuned topological transition of superconductivity in Dirac semimetal nanowire $\mathrm{Cd}_{3} \mathrm{As}_{2}$ Josephson junctions. A gatesensitive multiple-modes supercurrent interference pattern due to quantized surface subbands exists in the electron branch, and a gate-independent supercurrent oscillation in the hole branch is observed due to the gapless nature of the surface band. The critical magnetic field $B_{c}$ and critical supercurrent $I_{c}$ are both enhanced in the hole conduction branch, suggesting the topology enhanced superconductivity. Such gate-controlled topological transition of superconductivity is consistent with the previous observations of the presence (absence) of Majorana zero modes in the hole (electron) branch in such a similar nanowire system [25]. Our results provide a route to use $\mathrm{Cd}_{3} \mathrm{As}_{2}$ nanowire as a platform for generating and manipulating Majorana bound states, which is compatibly applicable to future networks design of braiding operations.

This work was supported by National Key Research and Development Program of China (No. 2018YFA0703703 and No. 2016YFA0300802), National Natural Science Foundation of China (No. 91964201, No. 61825401, and No. 11774004), and financially supported by Netherlands Organization for Scientific Research (NWO) through a VENI grant (680-47-463) and a VECI grant.

\footnotetext{
${ }^{*}$ C.-Z. L. and A.-Q. W. contributed equally to this work. †chuan.li@utwente.nl

†liaozm@pku.edu.cn
}

[1] T. M. Rice and M. Sigrist, $\mathrm{Sr}_{2} \mathrm{RuO}_{4}$ : An electronic analogue of ${ }^{3} \mathrm{He}$ ? J. Phys. Condens. Matter 7, L643 (1995).

[2] K. Ishida, H. Mukuda, Y. Kitaoka, K. Asayama, Z. Q. Mao, Y. Mori, and Y. Maeno, Spin-triplet superconductivity in $\mathrm{Sr}_{2} \mathrm{RuO}_{4}$ identified by ${ }^{17} \mathrm{O}$ Knight shift, Nature (London) 396, 658 (1998).

[3] G. M. Luke, Y. Fudamoto, K. M. Kojima, M. I. Larkin, J. Merrin, B. Nachumi, Y. J. Uemura, Y. Maeno, Z. Q. Mao, Y. Mori, H. Nakamura, and M. Sigrist, Time-reversal symmetry-breaking superconductivity in $\mathrm{Sr}_{2} \mathrm{RuO}_{4}$, Nature (London) 394, 558 (1998).

[4] L. Fu and C. L. Kane, Superconducting Proximity Effect and Majorana Fermions at the Surface of a Topological Insulator, Phys. Rev. Lett. 100, 096407 (2008).

[5] R. M. Lutchyn, J. D. Sau, and S. Das Sarma, Majorana Fermions and a Topological Phase Transition in Semiconductor-Superconductor Heterostructures, Phys. Rev. Lett. 105, 077001 (2010).

[6] Y. Oreg, G. Refael, and F. von Oppen, Helical Liquids and Majorana Bound States in Quantum Wires, Phys. Rev. Lett. 105, 177002 (2010).

[7] S. Nadj-Perge, I. K. Drozdov, J. Li, H. Chen, S. Jeon, J. Seo, A. H. MacDonald, B. A. Bernevig, and A. Yazdani, Observation of Majorana fermions in ferromagnetic atomic chains on a superconductor, Science 346, 602 (2014).

[8] B. E. Feldman, M. T. Randeria, J. Li, S. Jeon, Y. Xie, Z. Wang, and I. K. Drozdov, B. Andrei Bernevig, and A. Yazdani, High-resolution studies of the Majorana atomic chain platform, Nat. Phys. 13, 286 (2017).

[9] J. X. Yin, Z. Wu, J. H. Wang, Z. Y. Ye, J. Gong, X. Y. Hou, L. Shan, A. Li, X. J. Liang, X. X. Wu, J. Li, C. S. Ting, Z. Q. Wang, J. P. Hu, P. H. Hor, H. Ding, and S. H. Pan, Observation of a robust zero-energy bound state in iron-based superconductor Fe(Te,Se), Nat. Phys. 11, 543 (2015).

[10] D. Wang, L. Kong, P. Fan, H. Chen, S. Zhu, W. Liu, L. Cao, Y. Sun, S. Du, J. Schneeloch, R. Zhong, G. Gu, L. Fu, H. Ding, and H.-J. Gao, Evidence for Majorana bound states in an iron-based superconductor, Science 362, 333 (2018).

[11] P. Zhang, K. Yaji, T. Hashimoto, Y. Ota, T. Kondo, K. Okazaki, Z. Wang, J. Wen, G. D. Gu, H. Ding, and S. Shin, Observation of topological superconductivity on the surface of an iron-based superconductor, Science 360, 182 (2018).

[12] T. Machida, Y. Sun, S. Pyon, S. Takeda, Y. Kohsaka, T. Hanaguri, T. Sasagawa, and T. Tamegai, Zero-energy vortex bound state in the superconducting topological surface state of $\mathrm{Fe}(\mathrm{Se}, \mathrm{Te})$, Nat. Mater. 18, 811 (2019).

[13] V. Mourik, K. Zuo, S. M. Frolov, S. R. Plissard, E. P. A. M. Bakkers, and L. P. Kouwenhoven, Signatures of Majorana fermions in hybrid superconductor-semiconductor nanowire devices, Science 336, 1003 (2012).

[14] A. Das, Y. Ronen, Y. Most, Y. Oreg, M. Heiblum, and H. Shtrikman, Zero-bias peaks and splitting in an Al-InAs nanowire topological superconductor as a signature of Majorana fermions, Nat. Phys. 8, 887 (2012).

[15] M. T. Deng, C. L. Yu, G. Y. Huang, M. Larsson, P. Caroff, and H. Q. Xu, Anomalous zero-bias conductance peak in a $\mathrm{Nb}-\mathrm{InSb}$ nanowire-Nb hybrid device, Nano Lett. 12, 6414 (2012). 
[16] F. Pientka, A. Keselman, E. Berg, A. Yacoby, A. Stern, and B. I. Halperin, Topological Superconductivity in a Planar Josephson Junction, Phys. Rev. X 7, 021032 (2017).

[17] A. Fornieri, A. M. Whiticar, F. Setiawan, E. Portolés, A. C. C. Drachmann, A. Keselman, S. Gronin, C. Thomas, T. Wang, R. Kallaher, G. C. Gardner, E. Berg, M. J. Manfra, A. Stern, C. M. Marcus, and F. Nichele, Evidence of topological superconductivity in planar Josephson junctions, Nature (London) 569, 89 (2019).

[18] H. Ren, F. Pientka, S. Hart, A. T. Pierce, M. Kosowsky, L. Lunczer, R. Schlereth, B. Scharf, E. M. Hankiewicz, L. W. Molenkamp, B. I. Halperin, and A. Yacoby, Topological superconductivity in a phase-controlled Josephson junction, Nature (London) 569, 93 (2019).

[19] F. Setiawan, A. Stern, and E. Berg, Topological superconductivity in planar Josephson junctions: Narrowing down to the nanowire limit, Phys. Rev. B 99, 220506(R) (2019).

[20] J.-P. Xu, M.-X. Wang, Z. L. Liu, J.-F. Ge, X. Yang, C. Liu, Z. A. Xu, D. Guan, C. L. Gao, D. Qian, Y. Liu, Q.-H. Wang, F.-C. Zhang, Q.-K. Xue, and J.-F. Jia, Experimental Detection of a Majorana Mode in the core of a Magnetic Vortex inside a Topological Insulator-Superconductor $\mathrm{Bi}_{2} \mathrm{Te}_{3} / \mathrm{NbSe}_{2}$ Heterostructure, Phys. Rev. Lett. 114, 017001 (2015).

[21] H.-H. Sun, K.-W. Zhang, L.-H. Hu, C. Li, G.-Y. Wang, H.-Y. Ma, Z.-A. Xu, C.-L. Gao, D.-D. Guan, Y.-Y. Li, C. Liu, D. Qian, Y. Zhou, L. Fu, S.-C. Li, F.-C. Zhang, and J.-F. Jia, Majorana Zero Mode Detected with Spin Selective Andreev Reflection in the Vortex of a Topological Superconductor, Phys. Rev. Lett. 116, 257003 (2016).

[22] J. Wiedenmann, E. Bocquillon, R. S. Deacon, S. Hartinger, O. Herrmann, T. M. Klapwijk, L. Maier, C. Ames, C. Brune, C. Gould, A. Oiwa, K. Ishibashi, S. Tarucha, H. Buhmann, and L. W. Molenkamp, $4 \pi$-periodic Josephson supercurrent in HgTe-based topological Josephson junctions, Nat. Commun. 7, 10303 (2016).

[23] E. Bocquillon, R. S. Deacon, J. Wiedenmann, P. Leubner, T. M. Klapwijk, C. Brune, K. Ishibashi, H. Buhmann, and L. W. Molenkamp, Gapless Andreev bound states in the quantum spin Hall insulator HgTe, Nat. Nanotechnol. 12, 137 (2017).

[24] C. Li, J. C. de Boer, B. de Ronde, S. V. Ramankutty, E. van Heumen, Y. Huang, A. de Visser, A. A. Golubov, M. S. Golden, and A. Brinkman, 4 $\pi$-periodic Andreev bound states in a Dirac semimetal, Nat. Mater. 17, 875 (2018).

[25] A.-Q. Wang, C.-Z. Li, C. Li, Z.-M. Liao, A. Brinkman, and D.-P. Yu, $4 \pi$ Periodic Supercurrent from Surface States in $\mathrm{Cd}_{3} \mathrm{As}_{2}$ Nanowire-Based Josephson Junctions, Phys. Rev. Lett. 121, 237701 (2018).

[26] W. Yu, W. Pan, D. L. Medlin, M. A. Rodriguez, S. R. Lee, Z.-q. Bao, and F. Zhang, $\pi$ and $4 \pi$ Josephson Effects Mediated by a Dirac Semimetal, Phys. Rev. Lett. 120, 177704 (2018).

[27] Z. Lyu, Y. Pang, J. Wang, G. Yang, J. Fan, G. Liu, Z. Ji, X. Jing, C. Yang, F. Qu, and L. Lu, Protected gap closing in Josephson junctions constructed on $\mathrm{Bi}_{2} \mathrm{Te}_{3}$ surface, Phys. Rev. B 98, 155403 (2018).

[28] G. Yang, Z. Lyu, J. Wang, J. Ying, X. Zhang, J. Shen, G. Liu, J. Fan, Z. Ji, X. Jing, F. Qu, and L. Lu, Protected gap closing in Josephson trijunctions constructed on $\mathrm{Bi}_{2} \mathrm{Te}_{3}$, Phys. Rev. B 100, 180501(R) (2019).

[29] S. Cho, B. Dellabetta, R. Zhong, J. Schneeloch, T. Liu, G. Gu, M.J. Gilbert, and N. Mason, Aharonov-Bohm oscillations in a quasi-ballistic three-dimensional topological insulator nanowire, Nat. Commun. 6, 7634 (2015).

[30] L. A. Jauregui, M. T. Pettes, L. P. Rokhinson, L. Shi, and Y. P. Chen, Magnetic field-induced helical mode and topological transitions in a topological insulator nanoribbon, Nat. Nanotechnol. 11, 345 (2016).

[31] H. Pan, M. Wu, Y. Liu, and S. A. Yang, Electric control of topological phase transitions in Dirac semimetal thin films, Sci. Rep. 5, 14639 (2015).

[32] J. L. Collins, A. Tadich, W. Wu, L. C. Gomes, J. N. B. Rodrigues, C. Liu, J. Hellerstedt, H. Ryu, S. Tang, S.-K. Mo, S. Adam, S. A. Yang, M. S. Fuhrer, and M.T. Edmonds, Electric-field-tuned topological phase transition in ultrathin $\mathrm{Na}_{3} \mathrm{Bi}$, Nature (London) 564, 390 (2018).

[33] J. Liu, T. H. Hsieh, P. Wei, W. Duan, J. Moodera, and L. Fu, Spin-filtered edge states with an electrically tunable gap in a two-dimensional topological crystalline insulator, Nat. Mater. 13, 178 (2014).

[34] D. Shao, J. Ruan, J. Wu, T. Chen, Z. Guo, H. Zhang, J. Sun, L. Sheng, and D. Xing, Strain-induced quantum topological phase transitions in $\mathrm{Na}_{3} \mathrm{Bi}$, Phys. Rev. B 96, 075112 (2017).

[35] T. Nie, L. Meng, Y. Li, Y. Luan, and J. Yu, Phase transition studies of $\mathrm{Na}_{3} \mathrm{Bi}$ system under uniaxial strain, J. Phys. Condens. Matter 30, 125502 (2018).

[36] H. Wang, H. Wang, H. Liu, H. Lu, W. Yang, S. Jia, X.-J. Liu, X.C. Xie, J. Wei, and J. Wang, Observation of superconductivity induced by a point contact on 3D Dirac semimetal $\mathrm{Cd}_{3} \mathrm{As}_{2}$ crystals, Nat. Mater. 15, 38 (2016).

[37] L. Aggarwal, A. Gaurav, G. S. Thakur, Z. Haque, A. K. Ganguli, and G. Sheet, Unconventional superconductivity at mesoscopic point contacts on the 3D Dirac semimetal $\mathrm{Cd}_{3} \mathrm{As}_{2}$, Nat. Mater. 15, 32 (2016).

[38] L. He, Y. Jia, S. Zhang, X. Hong, C. Jin, and S. Li, Pressureinduced superconductivity in the three-dimensional topological Dirac semimetal $\mathrm{Cd}_{3} \mathrm{As}_{2}$, npj Quantum Mater. 1, 16014 (2016).

[39] C.-Z. Li, L.-X. Wang, H. Liu, J. Wang, Z.-M. Liao, and D.-P. Yu, Giant negative magnetoresistance induced by the chiral anomaly in individual $\mathrm{Cd}_{3} \mathrm{As}_{2}$ nanowires, Nat. Commun. 6, 10137 (2015).

[40] See Supplemental Material at http://link.aps.org/ supplemental/10.1103/PhysRevLett.126.027001 for sample growth and transport measurements, details of supercurrent interference, finite momentum shift due to Zeeman effect, and results of device 2, which includes Refs. [41-42].

[41] D. Son and B. Spivak, Chiral anomaly and classical negative magnetoresistance of Weyl metals, Phys. Rev. B 88, 104412 (2013).

[42] C.-Z. Li, A.-Q. Wang, C. Li, W.-Z. Zheng, A. Brinkman, D.-P. Yu, and Z.-M. Liao, Reducing Electronic Transport Dimension to Topological Hinge States by Increasing Geometry Size of Dirac Semimetal Josephson Junctions, Phys. Rev. Lett. 124, 156601 (2020).

[43] M. Neupane, S. Y. Xu, R. Sankar, N. Alidoust, G. Bian, C. Liu, I. Belopolski, T. R. Chang, H.T. Jeng, H. Lin, A. Bansil, F. Chou, and M. Z. Hasan, Observation of a three- 
dimensional topological Dirac semimetal phase in highmobility $\mathrm{Cd}_{3} \mathrm{As}_{2}$, Nat. Commun. 5, 3786 (2014).

[44] S. Jeon, B. B. Zhou, A. Gyenis, B. E. Feldman, I. Kimchi, A. C. Potter, Q. D. Gibson, R. J. Cava, A. Vishwanath, and A. Yazdani, Landau quantization and quasiparticle interference in the three-dimensional Dirac semimetal $\mathrm{Cd}_{3} \mathrm{As}_{2}$, Nat. Mater. 13, 851 (2014).

[45] Enze Zhang, Yanwen Liu, Weiyi Wang, Cheng Zhang, Peng Zhou, Z.-G. Chen, J. Zou, and F. Xiu, Magnetotransport properties of $\mathrm{Cd}_{3} \mathrm{As}_{2}$ nanostructures, ACS Nano 9, 8843 (2015).

[46] L.-X. Wang, C.-Z. Li, D.-P. Yu, and Z.-M. Liao, AharonovBohm oscillations in Dirac semimetal $\mathrm{Cd}_{3} \mathrm{As}_{2}$ nanowires, Nat. Commun. 7, 10769 (2016).

[47] B.-C. Lin, S. Wang, L.-X. Wang, C.-Z. Li, J.-G. Li, D. Yu, and Z.-M. Liao, Gate-tuned Aharonov-Bohm interference of surface states in a quasiballistic Dirac semimetal nanowire, Phys. Rev. B 95, 235436 (2017).

[48] C.-Z. Li, C. Li, L.-X. Wang, S. Wang, Z.-M. Liao, A. Brinkman, and D.-P. Yu, Bulk and surface states carried supercurrent in ballistic Nb-Dirac semimetal $\mathrm{Cd}_{3} \mathrm{As}_{2}$ nanowire-Nb junctions, Phys. Rev. B 97, 115446 (2018).
[49] K. Flensberg, J. B. Hansen, and M. Octavio, Subharmonic energy-gap structure in superconducting weak links, Phys. Rev. B 38, 8707 (1988).

[50] C.-Z. Li, A.-Q. Wang, C. Li, W.-Z. Zheng, A. Brinkman, D.-P. Yu, and Z.-M. Liao, Fermi-arc supercurrent oscillations in Dirac semimetal Josephson junctions, Nat. Commun. 11, 1150 (2020).

[51] K. Gharavi, G. W. Holloway, C. M. Haapamaki, M. H. Ansari, M. Muhammad, R. R. LaPierre, and J. Baugh, Josephson interference due to orbital states in a nanowire proximity effect junction, arXiv:1405.7455.

[52] K. Gharavi and J. Baugh, Orbital Josephson interference in a nanowire proximity-effect junction, Phys. Rev. B 91, 245436 (2015).

[53] K. Zuo, V. Mourik, D. B. Szombati, B. Nijholt, D. J. van Woerkom, A. Geresdi, J. Chen, V. P. Ostroukh, A. R. Akhmerov, S. R. Plissard, D. Car, E. P. A. M. Bakkers, D. I. Pikulin, L. P. Kouwenhoven, and S. M. Frolov, Supercurrent Interference in Few-Mode Nanowire Josephson Junctions, Phys. Rev. Lett. 119, 187704 (2017).

[54] B.-C. Lin, S. Wang, A.-Q. Wang, Y. Li, R.-R. Li, K. Xia, D. $\mathrm{Yu}$, and Z.-M. Liao, Electric Control of Fermi Arc Spin Transport in Individual Topological Semimetal Nanowires, Phys. Rev. Lett. 124, 116802 (2020). 